OPERATIONAL EXPERIENCE WITH COMPRESSED GEOMETRY ACCELERATION TUBES IN THE OAK RIDGE 25URC TANDEM ACCELERATOR*

C. M. Jones, D. L. Haynes, R. C. Juras, M. J. Meigs, and N. F. Ziegler Oak Ridge National Laboratory P. O. Box 2008

Oak Ridge, Tennessee 37831-6368

CONF-8905141--2

ABSTRACT

DE89 014071

Installation of compressed geometry acceleration tubes and other associated modifications have increased the effective voltage capability of the Oak Ridge 25URC tandem accelerator by about 3 MV. Since mid-September 1988 , the accelerator has been operated routinely at terminal potentials up to $24 \mathrm{MV}$ and occasionally near $25 \mathrm{MV}$. In 3500 hours of full-column operation, including 1100 hours at potentials above 22 MV, no significant spark-induced damage was observed. Some considerations related to further improvements in voltage performance are discussed.

I. Introduction

The Oak Ridge 25URC tandem electrostatic accelerator ${ }^{1,2}$ is one of two accelerators operated by the Holifield Heavy ion Research Facility ${ }^{3}$ (HHIRF) at the Oak Ridge National Laboratory. Placed into routine service in 1982 , the accelerator has provided a wide range of heavy ion beams for research in nuclear and atomic physics. These beams have been used both directly and after further acceleration by the Oak Ridge Isochronous Cyclotron (ORIC). ${ }^{4}$

- Research sponsored by the U.S. Department of Energy under contract DE-ACO5. 840R21400 with Martin Marietta Energy Systems, Inc.

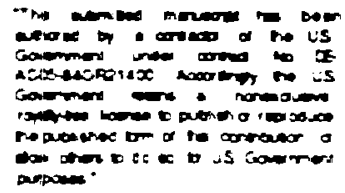


The 25URC Pelletron accelerator was manufactured by the National Electrostatics Corporation (NEC). One of two operating accelerators of its size in the world, it was the first large accelerator to be designed using a folded configuration. Although designed to orerate at terminal potentials up to $25 \mathrm{MV}$, its operation prior to the present modifications had been limited to $23.5 \mathrm{MV}$ for demonstrations and 22.0 MV for scheduled experiments. These limits were partially programmatic in origin, but were also related to limitations which appear to have been associated with the acceleration tube; most basically, a tendency to spark at higher terminal potentials.

While voltage performance of the accelerator had been adequate for the experimental program, it seemed clear that improvement in voltage performance could be of direct benefit to the experimental program in the future. Therefore, we began, in June 1986, a program of modifications and tests which was designed to improve voltage performance of the accelerator. The result of this program was replacement of the original acceleration tubes with tubes of a compressed geometry design. 5 In this design, which utilizes a modified NEC high-gradient $17-\mathrm{cm}-$ long tube section, the 3-cm-thick heatable aperture assembly provided as part of the original installation is replaced with an aperture assembly of essentially zero length. With this change, seven tube sections can be installed in the space previously occupied by six, thus increasing the effective insulator length per unit column length by a factor of $7 / 6=1.17$. The result of this increase in effective insulator length is expected to be an approximately corresponding fractional increase in voltage performance. A detailed discussion of the compressed geometry design and its implementation in the 25URC accelerator has been provided in Ref. 6 and the references cited therein. 
Installation of compressed geometry acceleration tubes in the 25URC accelerator and initial conditioning was completed in January 1988. This was followed by conditioning and tests in two 18-day periods in April-May 1988 and August-September 1988. At the end of the second period, pairs of units and individual units had been conditioned to an average stable gradient of approximately 1.04 MV/unit," and stable operation of the accelerator with beam, was demonstrated at 25.5 MV for one hour without sparks or tics. A few days after completion of these tests, the accelerator was operated for use in the experimental program at a terminal potential of 25.0 MV $€$ Subsequently, the accelerator has been operated routinely for the experimental program at potentials up to $24 \mathrm{MV}$ and occasionally at potentials near $25 \mathrm{MV}$. In this paper, we discuss some aspects of operation of the accelerator in the period September 17,1988, through April 30, 1989, with emphasis on the effects of operation at high potential.

\section{Operationil Experience}

The immediate practical effect of the compressed geometry acceleration tube installation has been to increase the voltage capability of the accelerator by approximately 3 MV. This improvement is graphically illustrated in Fig. 1 where we show terminal voltage distribution functions ior the period October 1,1985 , through September 30, 1986 (the fiscal year in which work on the compressed geometry acceleration tubes began! and the period September 17, 1988, through April 30. 1989. Specifically, the improved terminal voltage capability of the accelerator has enabled us to provide beams of energies and intensities which would not otherwise

\footnotetext{
"The configuration of the 25URC accelerator is based on $2761-\mathrm{cm}$-long live modules or "units" separated by two major and three minor dead sections.
} 
have been possible. Examples of such beams are $500 \mathrm{MeV} 90 \mathrm{Zr}$ (tandem-only operation) and $885 \mathrm{MeV} 156 \mathrm{Gd}$ (coupled operation). It is also of interest to note that of the 3500 hours of full-column operation in the period September 17, 1988, through April $30,1989,1100$ hours have been at potentials greater than 22 MV.

In keeping with our previous policy, we have continued to attempt to minimize the frequency of full-column sparks. Thus, in the period September 17, 1988, through April 30,1989 , only 33 full-column sparks occurred. A distribution function for the terminal voltage of these sparks is given in Fig. 2. As cari by seen, all but two sparks occurred at terminal voltages exceeding $20 \mathrm{MV}$. All of these sparks appear to be "tank" sparks, i.e., sparks which result in only minimal vacuum response in the acceleration tubes. There has been no evidence of spark-induced deconditioning or damage to the acceleration tubes, column structure, or corona points. On several occasions, sparks have resulted in minor damage to column electronic components, but this damage has not resulted in lost time or unscheduled maintenance. A problem with spark-induced damage to terminal chain inductor cables was solved by installation of cables and insulators of a new design.

An interesting question concerns the frequency of sparks as a function of terminal potential and tank gas pressure. The limited data which we have recorded in this period is shown in Figs. 3 and 4 . In these figures, we show the frequency of sparks, summed over $1 \mathrm{MV}$-intervals, for two tank gas pressures, 5.4 bar (65 psig) and 6.4 bar ( 80 psig). As can be seen, the observed spark frequency is in the order of 1 spark/100 hours, or less, except for the highest potentials. We suspect that the increased frequency observed at the highest potentials may not represent a fundamental limitation, but rather a lack of conditioning against full-column sparks as 
discussed below. It should be emphasized again that Figs. 3 and 4 represent a total of only 33 sparks, and thus, rather poor statistics.

III. Discussion

While it is clear that operation of the 25URC accelerator with compressed geometry acceleration tubes has resulted in a significant improvement in voltage capability, several interesting questions remain. First, what is an acceptable spark frequency for the 25URC accelerator? Since recovery from a full-column spark typically takes about 20 minutes, the present frequency of about one spark per hundred hours of operation has only a small effect on operation for most experiments. Clearly, the more important consideration is spark-induced damage. As noted above, no significant damage has been observed for the small number of sparks experienced to date. Our present plan is to continue conservative operation of the accelerator and gather more data on spark-induced damage before significantly increasing the spark rate. As an aside, we note that spark rate, rather than acceleration tube conditioning phenomena, is, with rare exceptions, the limiting factor in achievable voltage.

A second question concerns the origin of full-column sparks in situations where the operating gradient is less than the gradient to which the acceleration tubes have been conditioned in subsets of the full column. Stated in another way, do sparks which originate in column or terminal-to-tank discharges represent a significant fraction of the sparks which are observed during operation? At this time, we do not have a definitive answer to this question. 
A third question concerns conditioning with respect to full-column sparks; that is. does the occurrence of a full-column spark decrease the probability of future fullcolumn sparks? We are reasonably sure that the answer to this question is "yes." This conclusion is based on two observations. The first is our experience with long runs of 100 hours or more at high constant terminal potentials. A typical observation is that the accelerator may spark several times at the beginning of such a run and then operate throughout the remainder of the run with either no sparks or a markedly reduced spark frequency. A second observation was made during the column voltage tests performed in 1979 without acceleration tubes. ${ }^{7}$ As noted in Ref. 7, two kinds of sparks were observed, sparks whose properties appeared to be dominated by longitudinal column currents, i.e., column sparks, and sparks whose properties appeared to be dominated by the column-to-tank arc, i.e., tank sparks. In Fig. 5, we have plotted the terminal potentials of the sparks which occurred during the column voltage tests as a function of sequence and also have indicated the type of each spark (except for a few sparks, for which the appropriate data were unavailable). Examination of Fig. 5 shows that column sparks show no apparent evidence of conditioning while tank sparks at 5.1 bar (60 psig) and 7.1 bar (90 psig) show an apparent conditioning effect which averages about $1.3 \%$ increase per spark. Since we believe all sparks experienced during operation of the accelerator are, in this context, tank sparks, we believe that the conditioning phenomena observed in 1979 may also be present in current operation.

In summary, installation of compressed geometry acceleration tubes has resulted in an initial improvement of about $3 \mathrm{MV}$ in voltage performance of the 25URC accelerator during routine operation with no apparent detrimental effects. Further 
improvement, resulting from additional operating experience and additional conditioning and tests, is expected in the future.

Acknowiedgements

We wish to express our appreciation to the HHIRF operations staff, M. R. Dinehart, H. D. Hackler, C. L. Haley, C. A. Irizarry, C. T. LeCroy, S. N. Murray, B. K. Sizemore, and S. D. Taylor, for their skill and diligence during the operational period discussed in this paper.

References

1. J. K. Bair, J. A. Biggerstaff, C. M. Jones, J. D. Larson, J. W. McConnell, W. T. Milner, and N. F. Ziegler, IEEE Trans. Nucl. Sci. NS-22, No. 3 (1975) 1655.

2. C. M. Jones, Proc. Third Int. Conf. on Electrostatic Accelerator Technology, Oak Ridge, Tennessee (April 1981) p. 23.

3. C. M. Jones, G. D. Alton, J. B. Ball, J. A. Biggerstaff, D. T. Dowling, K. A. Erb, D. L. Haynes, D. E. Hoglund, E. D. Hudson, R. C. Juras, S. N. Lane, C. A. Ludemann, J. A. Martin, M. J. Meigs, S. W. Mosko, D. K. Olsen, and N. F. Ziegler, Nucl. Instrum. and Meth. in Phys. Res. A268 (1988) 308.

4. J. A. Martin, D. T. Dowling, D. L. Haynes, E. D. Hudson, C. M. Jones, R. C. Juras, S. N. Lane, C. A. Ludemann, M. J. Meigs, W. T. Milner, S. W. Mosko, D. K. Olsen, and N. F. Ziegler, Proc. Eleventh Int. Cont. on Cyclotrons and Their Applications, Tokyo, Japan (October 1986) p. 38.

5. W. Assmann, G. Korschinek, and H. Münzer, Nucl. Instrum. and Meth. in Phys. Res. 220 (1984) 86. 
6. C. M. Jones, D. L. Haynes, R. C. Juras, M. J. Meigs, N. F. Ziegler, J. E. Raatz, and R. D. Rathmell, Nucl. Instrum. and Meth. in Phys. Res. A276 (1989) 413.

7. C. M. Jones, Nucl. Instrum. and Meth. 184 (1981) 145. 
Figure Captions

1. The number of runs in 1-MV-wide intervals is shown as a function of terminal potential for the period October 1, 1985, through September 30, 1986 and the period September 17, 1988, through April 30, 1989. In this context, a run represents a new tune of the accelerator, i.e., a new beam energy or ion species.

2. The number of sparks in 1-MV-wide intervals is shown as a function of terminal potential for the time period September 17, 1988, through April 30, 1989.

During this period, the accelerator was operated in full-column mode for about 3500 hours.

3. The frequericy of sparks, summed over 1-MV-wide intervals, is shown as a function of terminal potential for the period September 17, 1988, through April 30, 1989, and an operating tank gas pressure of 5.4 bar (65 psig). The data shown is for a total of 16 sparks.

4. The frequency of sparks, summed over 1-MV-wide intervals, is shown as a function of terminal potential for the period September 17, 1988, through April 30, 1989, and an operating tank gas pressure of 6.4 bar (80 psig). The data shown is for a total of 17 sparks.

5. The terminal voltages of sparks which occurred during column voltage tests in 1979,7 without acceleration tubes, is shown as a function of sequence. Closed and open circles indicate sparks whose troperties appeared to be dominated by longitudinal column currents (column sparks), and sparks whose properties appeared to be dominated by the column-to-tank arc (tank sparks), respectively. $X ' s$ indicate sparks for which it was not possible to determine the breakdown mode. 


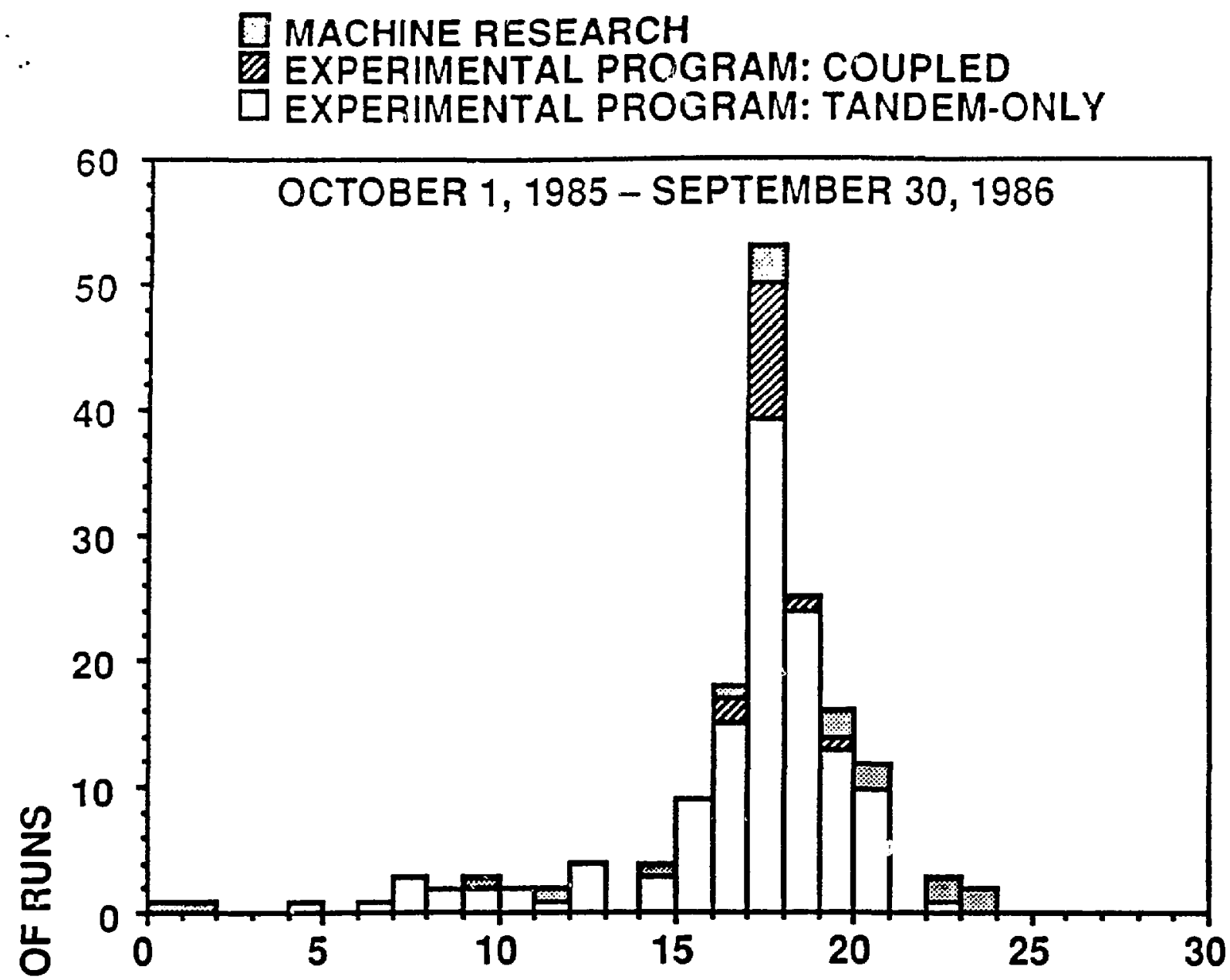

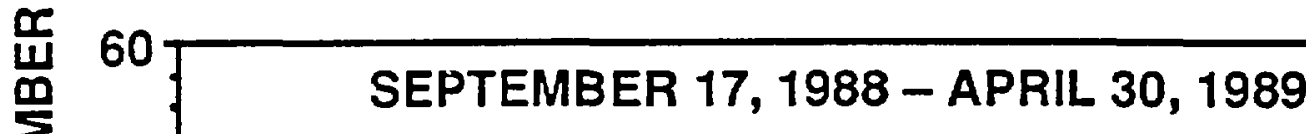

2

50

40

30

20

10

0.

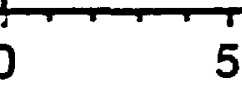

5

10

15

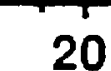

TERMINAL VOLTAGE (MV)

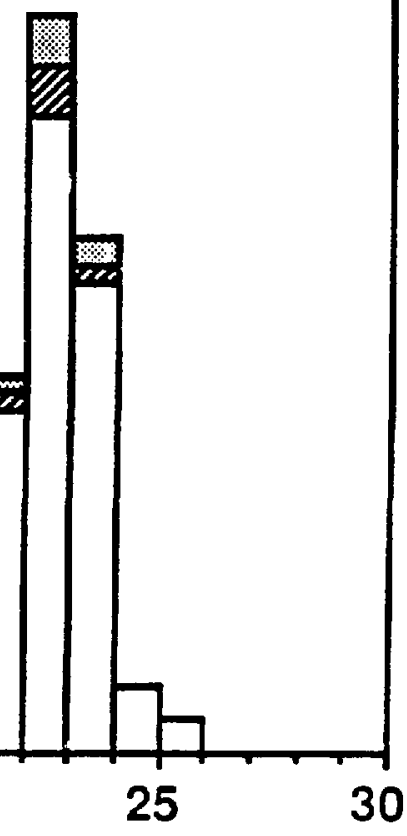


SPARK VOLTAGE DISTRIBUTION 9/17/88-4/30/89

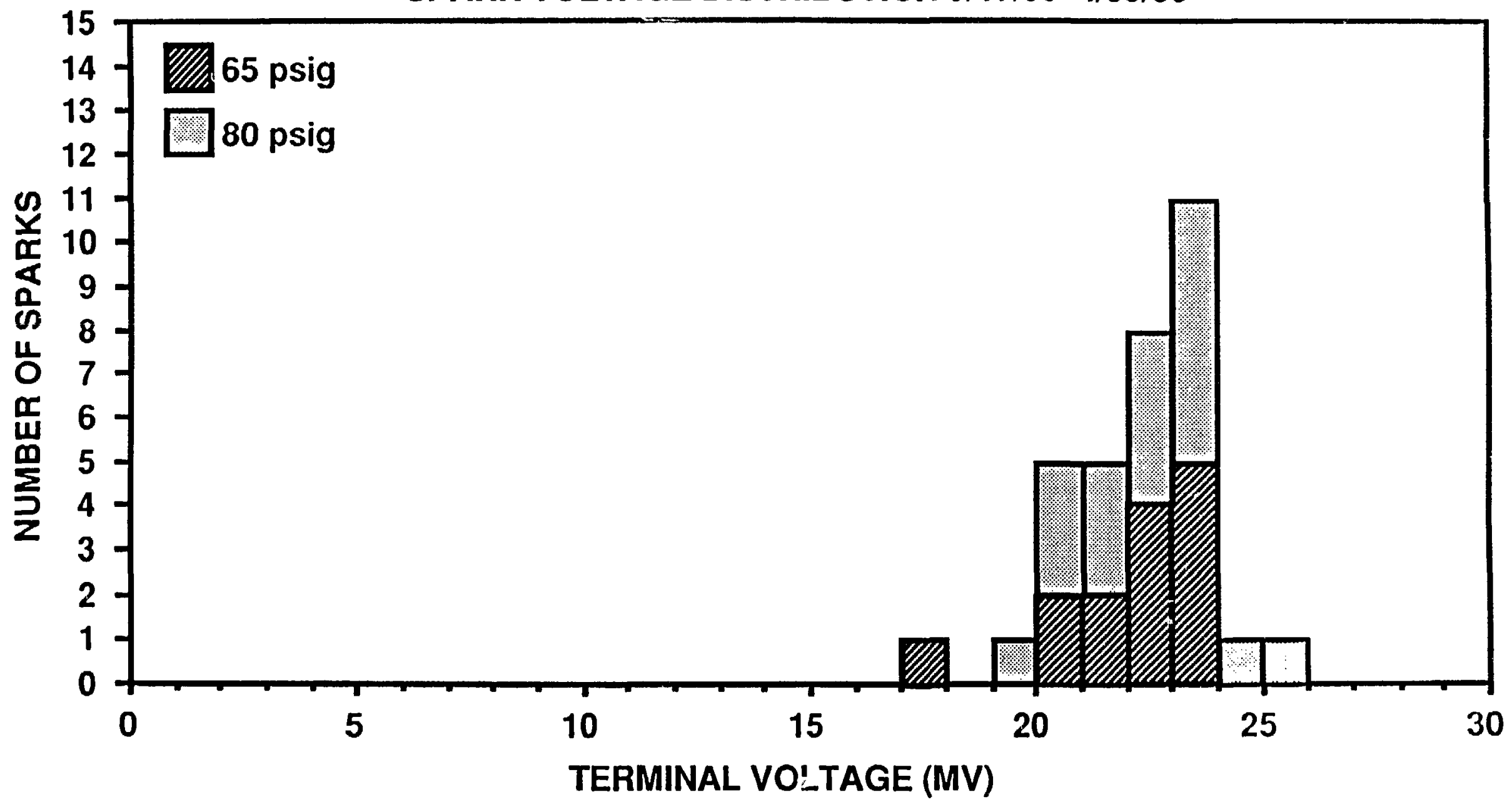

Fig. 2 
SPARK FREQUENCY 9/17/88-4/30/89

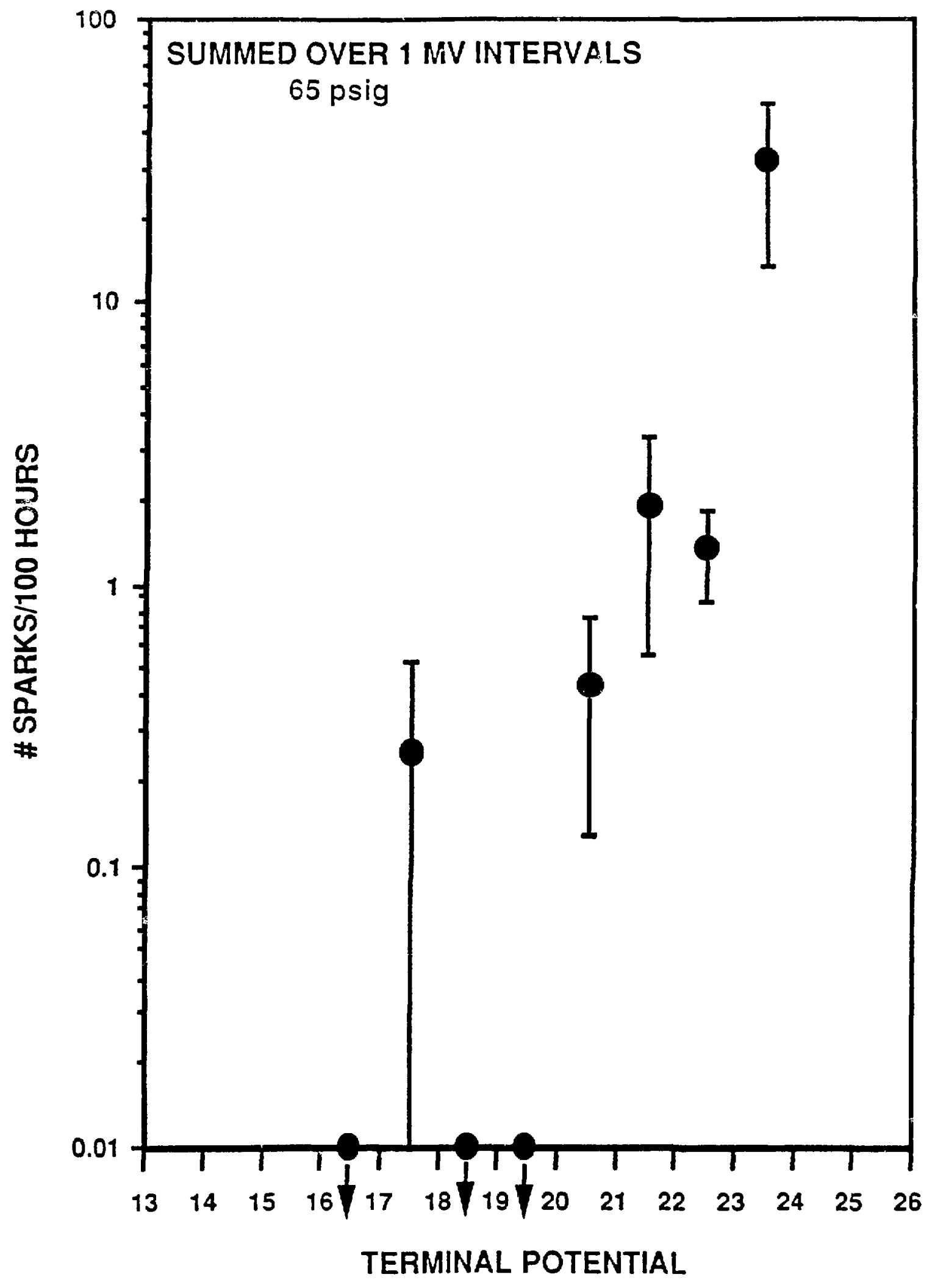

Fig. 3 


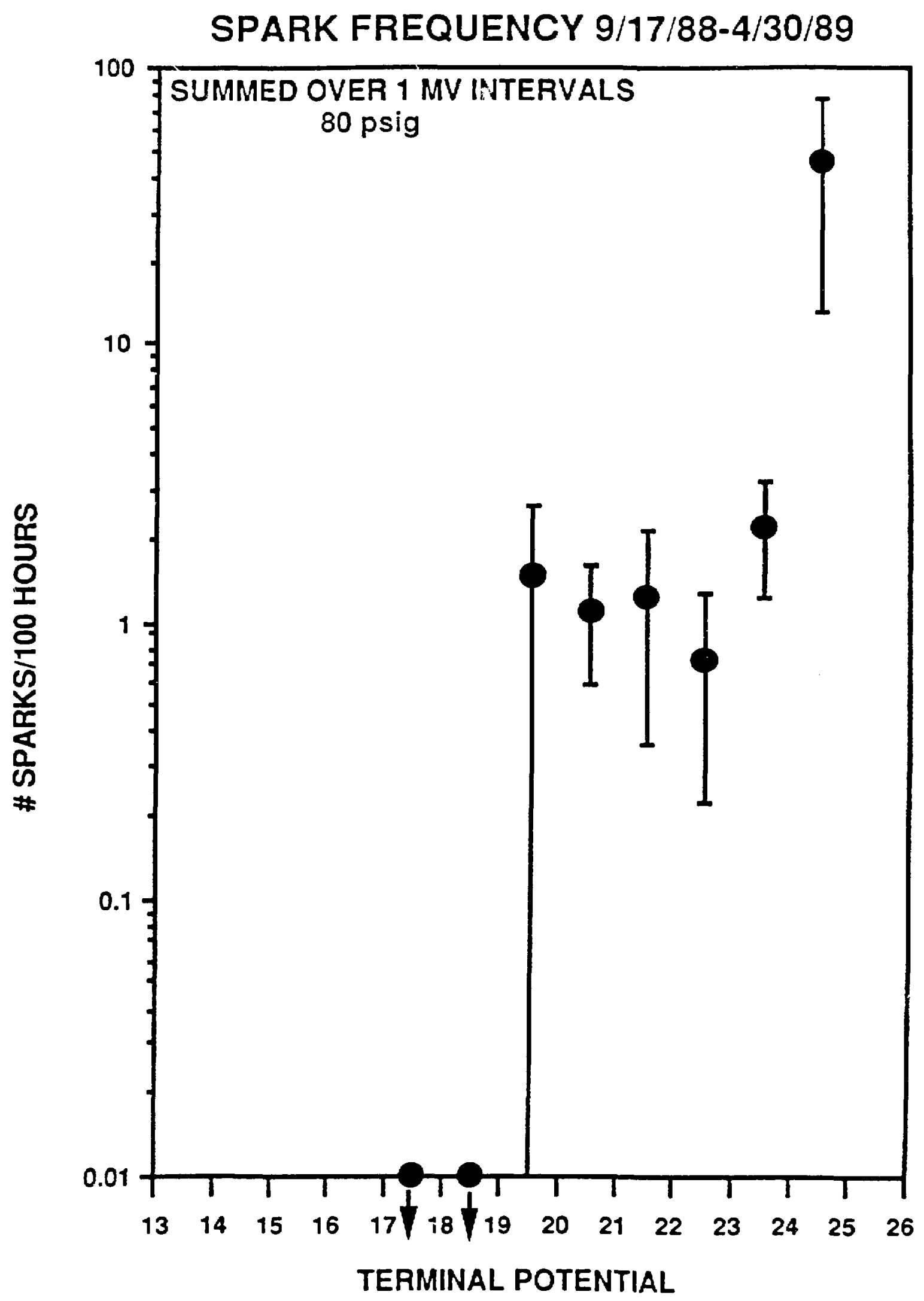

Fig. 4 


\section{COLUMN VOLTAGE TESTS - MAY 1979}

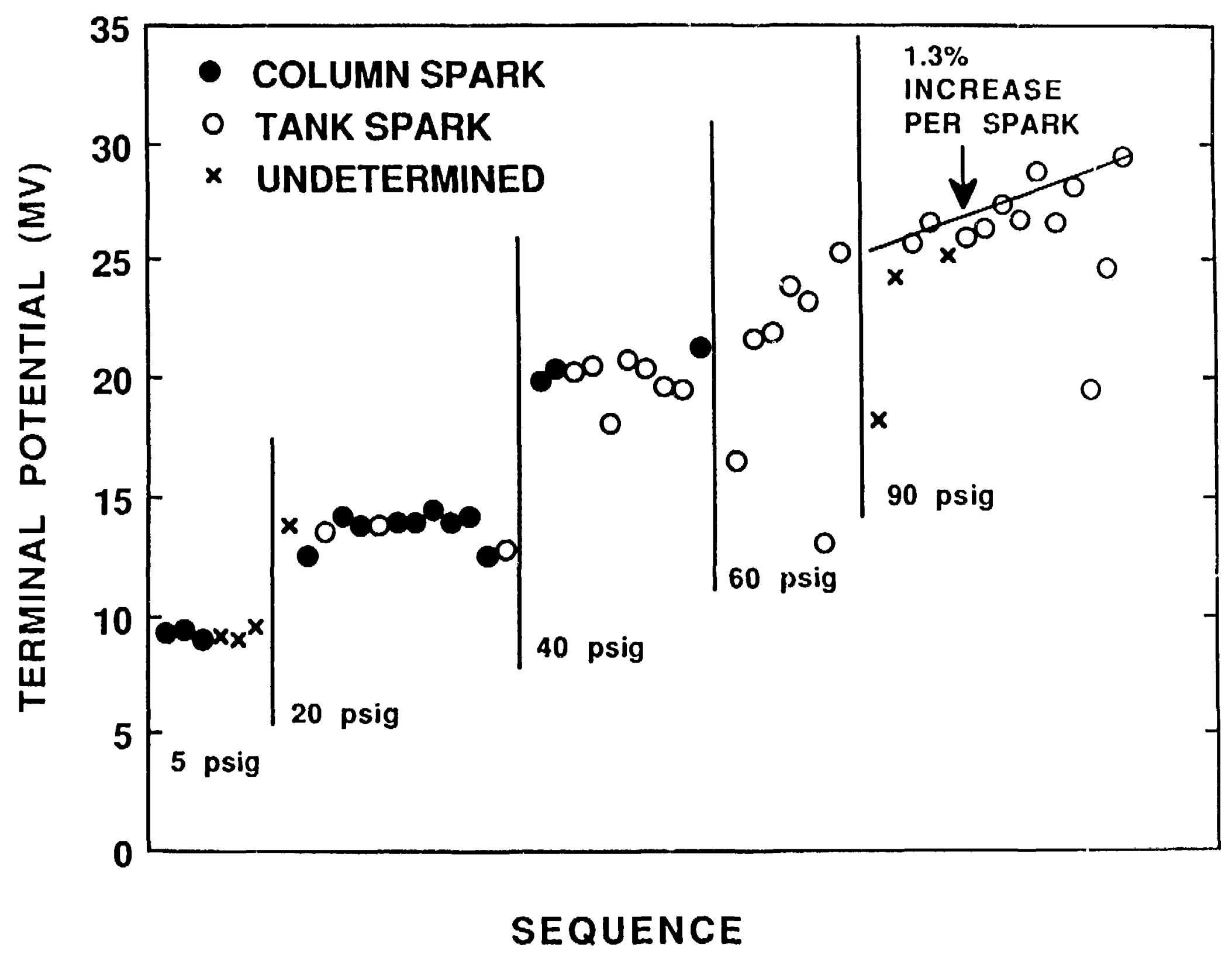

Fig. 5 